\title{
An XFEM/level set approach to modelling surface/interface effects and to computing the size-dependent effective properties of nanocomposites
}

\author{
Received: date / Accepted: date
}

\begin{abstract}
In a nanostructured material, the interfaceto-volume ratio is so high that the interface energy, which is usually negligible with respect to the bulk energy in solid mechanics, can no longer be neglected. The interfaces in a number of nanomaterials can be appropriately characterized by the coherent interface model. According to the latter, the displacement vector field is continuous across an interface in a medium while the traction vector field across the same interface is discontinuous and must satisfy the Laplace-Young equation. The present work aims to elaborate an efficient numerical approach to dealing with the interface effects described by the coherent interface model and to determining the size-dependent effective elastic moduli of nanocomposites. To achieve this twofold objective, a computational technique combining the level set method and the extended finite element method is developed and implemented. The numerical results obtained by the developed computational technique in the two-dimensional context are compared and discussed with respect to the relevant exact analytical solutions used as benchmarks. The computational technique elaborated in the present work is expected to be an efficient tool for evaluating the overall sizedependent elastic behaviour of nanomaterials and nanosized structures.
\end{abstract}

Keywords Nanomaterials · Extended Finite Element Method · Level-set · Microstructures · Percolation · Multiple Level-Sets

\section{Introduction}

Nanostructured materials and systems are of fundamental interest. Indeed, as compared with their microstruc-

J. Yvonnet

Université Paris-Est, Laboratoire de Mécanique (LAMEA2545), 5 Bd Descartes, F-77454 Marne-la-Vallée Cedex 2, France

Tel.: 0033150957795

Fax: 0033150957799

E-mail: jyvonnet@univ-mlv.fr tured counterparts, they often exhibit superior mechanical and physical performances [1]. The main tool currently used to study the mechanical behaviour of nanomaterials and nanosystems is the molecular dynamics (MD) simulation $[2 ; 3 ; 4]$, which is suitable for studying objects consisting of only a few atoms or when the phenomena under investigation occur at a so small length scale that the standard framework of continuum mechanics is no longer valid. At the same time, there is a range of fine scales where the number of atoms involved is relatively important, so that the mechanical fields of interest are smooth enough to fall within the scope of continuum mechanics upon taking into account additional effects which are negligible at the usual macroscopic scale. Such enriched continuum approaches have the definite advantage of avoiding the treatment of large clusters of atoms, which is computationally expensive, and of preserving the applicableness of widespread powerful numerical techniques such as the finite element method (FEM). One of the non-classical effects which should be taken into account in the continuum mechanics modelling of nanomaterials and nanostructures is the interface (or surface) effect. In fact, the high interface-tovolume ratio of a nanomaterial or nanostructure makes its interface energy comparable to or dominant over its bulk energy. One consequence of this fact is that the overall behaviour of a nanomaterial or nanostructure becomes size-dependent $[5 ; 6]$. The objective of the present work is twofold. First, it has the purpose of elaborating an efficient computational approach to modelling surface/interface effects by combining the extended finite element method (XFEM) and the level set method (LSM). Second, it aims to apply the elaborated computational approach to determine the size-dependent effective elastic properties of a composite made of a matrix reinforced by nanoparticles or nanofibers.

A wide range of interface effects can be suitably described by imperfect interface models. By definition, an interface in a medium is perfect if both the displacement and traction vector fields across it are continuous; otherwise, it is said to be imperfect. Among a great number 
of imperfect interface models constructed and justified to account for different interface effects [7], the coherent and spring-layer interface models are the two most important ones and also the mostly widely used ones. In the coherent interface model, the displacement vector field is continuous across an interface while the traction vector field across the same interface is discontinuous and has to verify the Laplace-Young equation [5]. In the spring-layer interface model [8], the traction vector field is continuous across an interface and proportional to the jump of the displacement vector field across the same interface. In the present work, the coherent interface model is adopted since it is appropriate for modelling the interface effect in nanomaterials $[9 ; 10 ; 11 ; 12]$.

Different works using the coherent interface model have recently been carried out to estimate the overall elastic properties of nanocomposites and nano-sized structural elements. In $[9 ; 10 ; 13 ; 14]$, Sharma and Ganti extended Eshelby's original formalism to nano-inclusions and obtained the closed-form expressions of Eshelby's tensor for spherical and circular cylindrical nano-inclusions. Le Quang and He proposed an extended version of the classical generalized self-consistent method to determine the size-dependent effective thermoelastic properties of nanocomposites with cylindrical and spherically anisotrop phases $[15 ; 16]$. In [12] Duan et al. derived closed-form expressions for the bulk and shear moduli as functions of the interface properties, and analyzed the dependence of the elastic moduli on the size of the inhomogeneities. Chen et al. [11] showed that exact size-dependent connections exist between the overall elastic moduli of unidirectional nanocomposites. Dingreville et al. have developed a framework to calculate analytically the sizedependent overall properties of nano-sized structural elements [17]. In all the works reported in the literature and aiming at determining analytically the overall properties of nanomaterials and nano-sized structural elements, the shapes of nano inhomogeneities and structural elements are required to be very simple and the sizes of nano inhomogeneities have to be uniform. The computational approach elaborated in this paper is a general one, which has not these limitations.

In [18], Gao et al. proposed a finite element method using surface elements to take into account for the surface effects. Nevertheless, a major concern in dealing with a strongly inhomogeneous material is the generation of a mesh matching the interfaces. Within the standard framework of FEM and especially in the 3D case, such an operation is numerically very difficult when inhomogeneities are numerous or/and of arbitrary shapes. To avoid this burden, the present work uses the levelset method [19] to describe every interface in a regular mesh as the zero-level set of a scalar field. In implementing the coherent interface model, the term associated to the traction vector jump is then introduced by enriching the finite element approximation with discontinuous functions constructed on the level-set basis. This novel way of dealing numerically with imperfect interfaces is reminiscent of the XFEM proposed by Belytschko and Black [20], Moës et al. [21] and Sukumar et al. [22; 23] to treat cracks and perfect interfaces. However, the coherent imperfect interfaces treated in our work are rather different from cracks in nature. First from the mechanical point of view, the imperfect interfaces studied by us are characterized by the continuity of the displacement vector (which implies the continuity of the surface strains owing to Hadamard's theorem) and the jump of the traction vector which is governed by the YoungLaplace equations; the cracks as treated in previous paper on XFEM are characterized by by the discontinuity of the displacement vector and the discontinuity of the tractions. From the numerical standpoint, the imperfect interfaces treated in our work give rise to a contribution to the stiffness matrix while this contribution is absent in the case of cracks. To our knowledge, it is the first time that the coherent imperfect interface is treated by XFEM/level-set.

The paper is organized as follows. In section 2, a curved interface is geometrically defined as a level set and some projection and differential operators associated to the interface are introduced in a coordinate-free way. In section 3 , we consider a solid made of a linearly elastic inhomogeneous material with the interfaces being described by the coherent interface model. The local equations governing the boundary value problem of the solid are first specified and a variational formulation of the problem suitable for a finite element approximation is then provided. Due to the coherent interfaces between the matrix and inhomogeneities, an additional stiffness term appears. In section 4 , we present the discretisation technique combining level-set method and XFEM. For simplicity, the technique is specified and implemented in the two-dimensional context and more precisely for plane strain and axisymmetric problems. In section 5, the numerical approach elaborated in the previous sections is applied to computing the size-dependent effective elastic properties of nanocomposites. A good few numerical examples are provided and compared with the relevant analytical exact solutions taken as benchmarks for testing the validity of the developed numerical approach. Further applications are illustrated for size-dependent overall properties of nanostructures with random distributions or shapes. In section 6 , a few concluding remarks are drawn.

\section{Geometric preliminaries}

Let $\Gamma_{I}$ be an interface between two neighbouring domains, which is taken to be a smooth $2 \mathrm{D}$ or $3 \mathrm{D}$ surface. A general method in differential geometry to describe such a smooth surface is to define it by an implicit function (see e.g. Do Carmo [24], Thorpe [25]). This method is in close relation with the level-set method 
[19], which is a numerical technique for tracking moving surfaces/interface and which is based on the idea of representing a surface as a level set of a higher dimension function. In the case of static interfaces, the two ideas coincide. In the present work we only consider static interfaces. In this context, $\Gamma_{I}$ is defined as the zero level-set of a function $\phi: \mathbb{R}^{d} \rightarrow \mathbb{R}:$

$$
\Gamma_{I}=\left\{\mathbf{x} \in \mathbb{R}^{d} \mid \phi(\mathbf{x})=0\right\},
$$

where $d$ is the dimension of the space under consideration. In the following, $\phi$ is assumed to be continuously differentiable and to have the property that $\nabla \phi(\mathbf{x}) \neq \mathbf{0}$ for all $\mathbf{x} \in \Gamma_{I}$. Thus, the unit normal vector field $\mathbf{n}(\mathbf{x})$ on $\Gamma_{I}$ is defined by

$$
\mathbf{n}(\mathbf{x})=\frac{\nabla \phi(\mathbf{x})}{\|\nabla \phi(\mathbf{x})\|},
$$

where $\|$.$\| denotes the Euclidian norm and \nabla($.$) denotes$ the gradient operator. Next, we introduce two orthogonal complementary projection operators:

$$
\mathbf{P}^{\perp}(\mathbf{x})=\mathbf{n}(\mathbf{x}) \otimes \mathbf{n}(\mathbf{x}), \quad \mathbf{P}(\mathbf{x})=\mathbf{I}-\mathbf{n}(\mathbf{x}) \otimes \mathbf{n}(\mathbf{x}) .
$$

where $\mathbf{I}$ is the second order unit tensor. Geometrically, $\mathbf{P}^{\perp}(\mathbf{x})$ describes the projection along the unit vector $\mathbf{n}(\mathbf{x})$ normal to $\Gamma_{I}$ at $\mathbf{x}$ while $\mathbf{P}$ characterizes the projection on the plane tangent to $\Gamma_{I}$ at $\mathbf{x}$.

Then, any vector function $\mathbf{w}$ admits the following decomposition:

$\mathbf{w}=\mathbf{w}_{n}+\mathbf{w}_{s}$,

with

$\mathbf{w}_{n}=\mathbf{P}^{\perp} \mathbf{w}, \quad \mathbf{w}_{s}=\mathbf{P} \mathbf{w}$.

Furthermore, when $\mathbf{w}$ is differentiable,

$\nabla \mathbf{w}=\nabla_{n} \mathbf{w}+\nabla_{s} \mathbf{w}, \nabla_{n} \mathbf{w}=\nabla \mathbf{w} \mathbf{P}^{\perp}, \nabla_{s} \mathbf{w}=\nabla \mathbf{w} \mathbf{P}$

Given any second-order tensor function $\mathbf{T}$, one defines its normal and tangential parts by

$$
\mathbf{T}_{n}=\mathbf{P}^{\perp} \mathbf{T} \mathbf{P}^{\perp}, \quad \mathbf{T}_{s}=\mathbf{P} \mathbf{T P} .
$$

Whenever $\mathbf{T}$ is differentiable, the divergence of $\mathbf{T}$ admits the following decomposition:

$$
\operatorname{div}(\mathbf{T})=\operatorname{div}_{n}(\mathbf{T})+\operatorname{div}_{s}(\mathbf{T}),
$$

where

$$
\operatorname{div}_{n}(\mathbf{T})=\nabla(\mathbf{T}): \mathbf{P}^{\perp}, \operatorname{div}_{s}(\mathbf{T})=\nabla(\mathbf{T}): \mathbf{P} .
$$

Wherever applicable, the indices $n$ and $s$ will denote normal and surface (tangential) components, respectively. It is worth noting that the operator $\mathbf{P}$ does not involve any basis change: the reader has to keep in mind that $\mathbf{w}_{s}$ and $\mathbf{T}_{s}$ denote the components of the projection of the associated vector and tensor, respectively, on the surface $\Gamma_{I}$. Thus $\mathbf{w}_{s}$ and $\mathbf{T}_{s}$ do not denote the components relative to the basis of the tangent plane to the surface. This projection tensor elegantly allows one to mix bulk and surface quantities in an equation.

\section{Governing equations}

In this section, we present the governing equations of linear elastostatics, together with the weak form and the discrete system for the $\mu$-XFEM.

\subsection{Strong form}

We consider a bodu which is described by an open bounded domain $\Omega \subset \mathbf{R}^{D}$, with boundary $\partial \Omega$. The boundary $\partial \Omega$ is composed of two disjoint complementary parts $\partial \Omega_{u}$ and $\partial \Omega_{t}$, where the Dirichlet (displacement) and Neumann (tractions) boundary conditions are prescribed, respectively. The field equations of elastostatics are:

$\operatorname{div}_{n}(\mathbf{T})=\nabla(\mathbf{T}): \mathbf{P}^{\perp}, \operatorname{div}_{s}(\mathbf{T})=\nabla(\mathbf{T}): \mathbf{P}$.

Without loss of generality, we assume that $\Gamma_{I}$ divides $\Omega$ into several domains $\Omega^{(i)}, i=1,2, \ldots, M$ such that $\Omega=\bigcup_{i=1, \ldots, M} \Omega^{(i)} \cup \Gamma_{I}, \bigcap_{i=1, \ldots, M} \Omega^{(i)}=\varnothing$. Let $\partial \Omega$ be the external boundary of $\Omega$ and $\partial \Omega^{(i)}$ the external boundary of each subdomain $\Omega^{(i)}$, such that $\partial \Omega^{(i)}=\partial \bar{\Omega}^{(i)} \cup \Gamma_{I}$. Let $\mathbf{n}^{(i)}$ the unit vector normal to $\partial \Omega^{(i)}$ pointing into $\Omega^{(i)}$. Thus, $\mathbf{n}^{(i)}$ is also the normal to $\Gamma_{I}$ pointing into the domain $\Omega^{(i)}$. For the sake of simplicity, we consider in the following only two domains. The equilibrium equations are then given by

$\operatorname{div}\left(\boldsymbol{\sigma}^{(i)}\right)+\mathbf{b}=\mathbf{0}$ in $\Omega^{(i)}$,

$\operatorname{div}_{s} \boldsymbol{\sigma}_{s}=-\llbracket \mathbf{t} \rrbracket=\left(\boldsymbol{\sigma}^{(2)}-\boldsymbol{\sigma}^{(1)}\right) \mathbf{n}^{(1)}$ on $\Gamma_{I}$.

In the above, $\boldsymbol{\sigma}$ denotes the bulk Cauchy stress tensor, b being a volumetric force term. Equation (11) is associated with bulk equilibrium, while Eq. (12) refers to the Laplace-Young equation resulting from the interface equilibrium. In particular, $\sigma_{s}$ is the surface stress tensor and $\operatorname{div}_{s}($.$) is defined in Eq. (10). The notation \llbracket . \rrbracket$ denotes the jump across $\Gamma_{I}$. The boundary conditions are described by

$\left\{\begin{array}{l}\boldsymbol{\sigma} \mathbf{n}=-\mathbf{F} \text { on } \partial \Omega_{F}, \\ \mathbf{u}=\overline{\mathbf{u}} \text { on } \partial \Omega_{u},\end{array}\right.$

where $\mathbf{F}$ and $\overline{\mathbf{u}}$ are prescribed tractions and displacements, respectively, and $\partial \Omega_{F}$ and $\partial \Omega_{u}$ are the Dirichlet and Neumann boundaries, respectively, such that $\partial \Omega=$ $\partial \Omega_{F} \cup \partial \Omega_{u}, \partial \Omega_{F} \cap \partial \Omega_{u}=\varnothing$.

According to the coherent interface model, the displacement jump across $\Gamma_{I}$ is null:

$\llbracket \mathbf{u} \rrbracket=\mathbf{0}$ on $\Gamma_{I}$,

The displacement vector is continuous across the interface but the strain tensor is discontinuous according to the Hadamard relation:

$\llbracket \boldsymbol{\epsilon} \rrbracket=\mathbf{a} \otimes \mathbf{n}+\mathbf{n} \otimes \mathbf{a}$,

where $\boldsymbol{\epsilon}$ is the infinitesimal strain tensor, and $\mathbf{a}$ is a realvalued vector. It follows from Eq. (15) that tangent or surface strains are continuous across $\Gamma_{I}$ :

$\llbracket \epsilon_{s} \rrbracket=\llbracket \mathbf{P} \epsilon \mathbf{P} \rrbracket=\mathbf{0}$. 

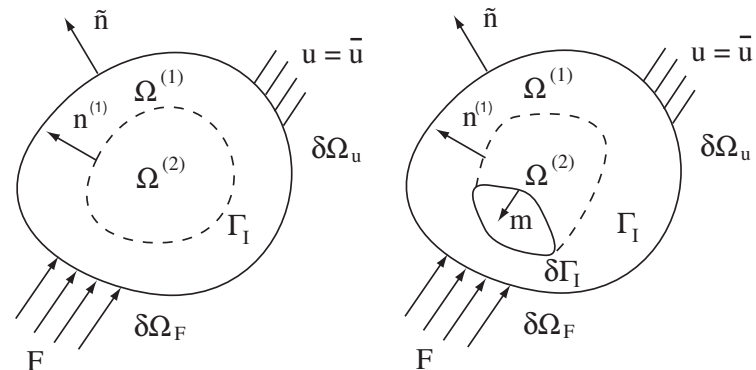

Fig. 1 (a) Closed interface $\Gamma_{I}$; (b) open interface $\Gamma_{I}$.

\subsection{Weak form}

The weak form associated with Eq. (11-13) is given in each domain as follows: that

Find $\mathbf{u} \in \mathcal{D}=\left\{\mathbf{u}=\overline{\mathbf{u}}\right.$ on $\left.\partial \Omega_{u}, \mathbf{u} \in H^{1}\left(\Omega^{(i)}\right)\right\}$, such

$\int_{\Omega^{(i)}} \boldsymbol{\sigma}^{(i)}(\mathbf{u}): \boldsymbol{\epsilon}^{(i)}(\boldsymbol{\delta} \mathbf{u}) d \Omega+\int_{\Gamma_{I}^{(i)}} \boldsymbol{\sigma}^{(i)} \mathbf{n}^{(i)} \cdot \boldsymbol{\delta} \mathbf{u} d \Gamma$

$-\int_{\Omega^{(i)}} \mathbf{b} \cdot \boldsymbol{\delta} \mathbf{u} d \Omega-\int_{\partial \bar{\Omega}_{F}^{(i)}} \mathbf{F} \cdot \boldsymbol{\delta} \mathbf{u} d \Gamma=0$

for all $\boldsymbol{\delta} \mathbf{u} \in H_{0}^{1}\left(\Omega^{(i)}\right)=\left\{\boldsymbol{\delta} \mathbf{u} \in H^{1}\left(\Omega^{(i)}\right), \boldsymbol{\delta} \mathbf{u}=\mathbf{0}\right.$ on $\left.\partial \Omega_{u}\right\}$ In the above, $\Gamma^{(i)}$ is the side of $\Gamma_{I}$ associated with the domain $\Omega^{(i)}$.

Adding the contributions of both the domains $\Omega^{(1)}$ and $\Omega^{(2)}$, and by using $\mathbf{n}^{(1)}=-\mathbf{n}^{(2)}$ and $\llbracket \boldsymbol{\delta} \mathbf{u} \rrbracket=\mathbf{0}$ on $\Gamma_{I}$, we obtain

$\int_{\Omega} \boldsymbol{\sigma}(\mathbf{u}): \boldsymbol{\epsilon}(\boldsymbol{\delta} \mathbf{u}) d \Omega+\int_{\Gamma_{I}}\left(\boldsymbol{\sigma}^{(1)}-\boldsymbol{\sigma}^{(2)}\right) \mathbf{n}^{(1)} \cdot \boldsymbol{\delta} \mathbf{u} d \Gamma=$

$=\int_{\Omega} \mathbf{b} \cdot \boldsymbol{\delta} \mathbf{u} d \Omega+\int_{\partial \Omega_{F}} \mathbf{F} \cdot \boldsymbol{\delta} \mathbf{u} d \Gamma$

Invoking the Laplace-Young equation, it yields

$\int_{\Omega} \boldsymbol{\sigma}(\mathbf{u}): \boldsymbol{\epsilon}(\boldsymbol{\delta} \mathbf{u}) d \Omega-\int_{\Gamma_{I}} \operatorname{div}_{s}\left(\boldsymbol{\sigma}_{s}(\mathbf{u})\right) \cdot \boldsymbol{\delta} \mathbf{u} d \Gamma=$

$\int_{\Omega} \mathbf{b} \cdot \boldsymbol{\delta} \mathbf{u} d \Omega+\int_{\partial \Omega_{F}} \mathbf{F} \cdot \boldsymbol{\delta} \mathbf{u} d \Gamma$

By using the relation:

$\operatorname{div}_{s}(\mathbf{T w})=\operatorname{div}_{s}\left(\mathbf{T}^{T}\right) \cdot \mathbf{w}+\mathbf{T}^{T}: \nabla_{s} \mathbf{w}$,

where $\mathbf{T}$ and $\mathbf{w}$ are continuously differentiable secondorder tensor and vector functions, respectively, we obtain, by the symmetry of $\boldsymbol{\sigma}_{s}$,

$$
\begin{aligned}
& \int_{\Gamma_{I}} \operatorname{div}_{s}\left(\boldsymbol{\sigma}_{s}\right) \cdot \boldsymbol{\delta} \mathbf{u} d \Gamma \\
& =\int_{\Gamma_{I}} \operatorname{div}_{s}\left(\boldsymbol{\sigma}_{s} \boldsymbol{\delta} \mathbf{u}\right) d \Gamma-\int_{\Gamma_{I}} \boldsymbol{\sigma}_{s}: \nabla_{s}(\boldsymbol{\delta} \mathbf{u}) d \Gamma .
\end{aligned}
$$

Due to the fact that $\mathbf{P}$ acts as the identity operator for $(.)_{s}$ quantities, we have:

$\operatorname{div}_{s}\left(\boldsymbol{\sigma}_{s} \boldsymbol{\delta} \mathbf{u}\right)=\operatorname{div}_{s}\left(\mathbf{P} \boldsymbol{\sigma}_{s} \mathbf{P} \boldsymbol{\delta} \mathbf{u}\right)$

$=\operatorname{div}_{s}\left(\mathbf{P} \boldsymbol{\sigma}_{s} \boldsymbol{\delta} \mathbf{u}_{s}\right)=\operatorname{div}_{s}\left(\boldsymbol{\sigma}_{s} \boldsymbol{\delta} \mathbf{u}_{s}\right)$

Applying Stokes' theorem, we obtain:

$\int_{\Gamma_{I}} \operatorname{div}_{s}\left(\boldsymbol{\sigma}_{s} \boldsymbol{\delta} \mathbf{u}_{s}\right) d \Gamma=\int_{\partial \Gamma_{I}} \boldsymbol{\sigma}_{s} \mathbf{m} \cdot \boldsymbol{\delta} \mathbf{u}_{s} d l$

$=\int_{\partial \Gamma_{I}} \hat{\mathbf{F}} \cdot \boldsymbol{\delta} \mathbf{u}_{s} d l=\int_{\partial \Gamma_{I}} \hat{\mathbf{F}} \cdot \mathbf{P} \boldsymbol{\delta} \mathbf{u} d l$

In the case where the interface $\Gamma_{I}$ is closed (see figure 1 (a)), the term (23) is equal to zero. Otherwise, for an open interface as depicted in figure $1(\mathrm{~b}), \partial \Gamma_{I}$ is the $(d-$ 2)-dimensional boundary of $\Gamma_{I}$ with $\mathbf{m}$ a unit outward vector normal to $\partial \Gamma_{I}$, and tangent to $\Gamma_{I}$ (see figure 1 (b)), $\hat{\mathbf{F}}$ being an applied force on $\partial \Gamma_{I}$.

Now, let us express the second term on the right-hand of expression (21). We have

$\boldsymbol{\sigma}_{s}: \nabla_{s}(\boldsymbol{\delta} \mathbf{u})=\left[\mathbf{P} \boldsymbol{\sigma}_{s} \mathbf{P}\right]: \nabla(\boldsymbol{\delta} \mathbf{u}) \mathbf{P}$.

Setting $[\nabla(\boldsymbol{\delta} \mathbf{u})]_{i j}=\nabla_{i j}$ and using the symmetry of $\mathbf{P}$ and the identity $\mathbf{P}^{2}=\mathbf{P}$, expression (24) can be written as:

$\left[P_{i k} \sigma_{k l}^{s} P_{l j}\right] \nabla_{i k} P_{k j}=\sigma_{k l}^{s}\left(P_{k i} \nabla_{i k} P_{k j} P_{j l}\right)=$

$=\boldsymbol{\sigma}_{s}:(\mathbf{P} \nabla(\boldsymbol{\delta} \mathbf{u}) \mathbf{P})=\boldsymbol{\sigma}_{s}: \frac{1}{2}\left(\mathbf{P} \nabla(\boldsymbol{\delta} \mathbf{u}) \mathbf{P}+[\mathbf{P} \nabla(\boldsymbol{\delta} \mathbf{u}) \mathbf{P}]^{T}\right)$

$=\sigma_{s}: \epsilon_{s}$

as $\boldsymbol{\sigma}_{s}$ is symmetric. Finally, the weak form is given by

$\int_{\Omega} \boldsymbol{\sigma}(\mathbf{u}): \boldsymbol{\epsilon}(\boldsymbol{\delta} \mathbf{u}) d \Omega+\int_{\Gamma_{I}} \boldsymbol{\sigma}_{s}(\mathbf{u}): \boldsymbol{\epsilon}_{s}(\boldsymbol{\delta} \mathbf{u}) d \Gamma$

$=\int_{\Omega} \mathbf{b} \cdot \boldsymbol{\delta} \mathbf{u} d \Omega+\int_{\partial \Omega_{F}} \mathbf{F} \cdot \boldsymbol{\delta} \mathbf{u} d \Gamma+\int_{\partial \Gamma_{I}} \hat{\mathbf{F}} \cdot \mathbf{P} \boldsymbol{\delta} \mathbf{u} d l$

\subsection{Constitutive equations}

Here we assume that the solid undergoes small displacements. In the context of a linear elastic model, the bulk constitutive law is given by:

$\boldsymbol{\sigma}(\mathbf{u})=\mathbb{C}^{(i)}:\left(\boldsymbol{\epsilon}(\mathbf{u})-\boldsymbol{\epsilon}^{*}\right)$,

where $\mathbb{C}^{(i)}$ is the fourth-order elastic stiffness tensor associated with domain $\Omega^{(i)}$ and $\epsilon^{*}$ is an eigenstrain prescribed on $\Omega^{*} \subset \Omega$.

According to [26], the surface stress $\boldsymbol{\sigma}_{s}$ is related to the surface-strain energy $\gamma$ by Schuttleworth's equation:

$\boldsymbol{\sigma}_{s}=\tau_{0} \mathbf{I}_{2}+\frac{\partial \gamma\left(\boldsymbol{\epsilon}_{s}\right)}{\partial \boldsymbol{\epsilon}_{s}}$ 
where $\tau_{0}$ is the strain-independent surface/interfacial tension and $\mathbf{I}_{2}$ is the unit tensor for surfaces. In the context of isotropic linear elastic interfaces, we have:

$$
\boldsymbol{\sigma}_{s}=\boldsymbol{\sigma}_{0}+\mathbb{C}^{s}: \boldsymbol{\epsilon}_{s}
$$

with

$$
C_{i j k l}^{s}=\lambda_{s} P_{i j} P_{k l}+\mu_{s}\left(P_{i k} P_{j l}+P_{i l} P_{j k}\right)
$$

where $\lambda_{s}$ and $\mu_{s}$ are Lamé's constants characterizing the interface $\Gamma_{I}$ and $\boldsymbol{\sigma}_{0}=\tau_{0} \mathbf{P}$. With this model, the weak form is finally given by:

$$
\int_{\Omega} \boldsymbol{\epsilon}(\boldsymbol{\delta} \mathbf{u}): \mathbb{C}: \boldsymbol{\epsilon}(\mathbf{u}) d \Omega+\int_{\Gamma_{I}} \mathbf{P} \boldsymbol{\epsilon}(\boldsymbol{\delta} \mathbf{u}) \mathbf{P}: \mathbb{C}^{s}: \mathbf{P} \boldsymbol{\epsilon}(\mathbf{u}) \mathbf{P} d \Gamma
$$$$
=\int_{\Omega} \boldsymbol{\delta} \mathbf{u} \cdot \mathbf{b} d \Omega+\int_{\partial \Omega_{F}} \boldsymbol{\delta} \mathbf{u} \cdot \mathbf{F} d \Gamma+\int_{\partial \Gamma_{I}} \mathbf{P} \boldsymbol{\delta} \mathbf{u} \cdot \hat{\mathbf{F}} d l
$$$$
+\int_{\Gamma_{I}} \mathbf{P} \boldsymbol{\epsilon}(\boldsymbol{\delta} \mathbf{u}) \mathbf{P}: \boldsymbol{\sigma}_{0} d \Gamma+\int_{\Omega} \boldsymbol{\epsilon}(\boldsymbol{\delta} \mathbf{u}): \mathbb{C}: \boldsymbol{\epsilon}^{*} d \Omega
$$

In [27], Chessa and Belytschko have taken into account surface tension with XFEM. In fluid mechanics, a surface tension, which can also be derived from a surface energy, is an isotropic surface stress tensor and must verify the classical Laplace equation. The surface energy involved in our paper is associated to a solid surface and its derivation with respect to the surface strain tensor gives a surface tensor which is in general not isotropic and must satisfy the Young Laplace equation. From a numerical point of view, in [27], the surface tension acts through en external forces energy term as it acts through an additional rigidity matrix in our work, as it will appear in the next section.

\section{Level-set/extended finite element discretization}

4.1 Evaluation of the unit vector normal to the interface

The domain $\Omega$ is discretized by $n$ nodes $n_{i}$ that do not necessarily match the interface $\Gamma_{I}$. Here we use a mesh of triangles, whereas other types of elements can be employed. Regular meshes can then be adopted for parallelepipedic domains, even if the interface has a complex geometrical shape.

In the present context, $\Gamma_{I}$ is defined as the zero levelset of a function $\phi(\mathbf{x})$, whose value is known at avery node $n_{i}$, i.e. $\phi\left(\mathbf{x}_{i}\right)=\phi_{i}$.

Wherever needed, the components of $\mathbf{n}(\mathbf{x})$ can be evaluated by:

$$
\mathbf{n}(\mathbf{x})=\frac{\nabla \tilde{\phi}(\mathbf{x})}{\|\nabla \tilde{\phi}(\mathbf{x})\|}
$$

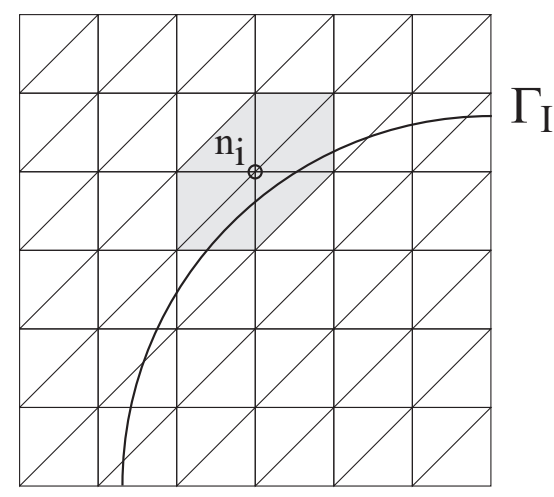

Fig. 2 Zero level-set of the function $\phi(\mathbf{x})$ and node $n_{i}$ whose support is cut by the interface $\Gamma_{I}$.

where

$$
\nabla \tilde{\phi}(\mathbf{x})_{i}=\sum_{j=1}^{n} \frac{\partial N_{j}(\mathbf{x})}{\partial x_{i}} \phi_{j}
$$

Here $N_{j}(\mathbf{x})$ are the standard finite element shape functions, $\phi_{j}$ are the nodal value of the level-set function, and $n$ is the number of nodes of the elements. In the present paper, we use simple linear finite element shape functions, though higher-order shape functions can be used [28], this interpolation being independent of the discretization of the weak form (30). The above approximation for $\mathbf{n}$ can then be used to evaluate the components of the projector $\mathbf{P}$ needed in the weak form (30).

\subsection{Discrete system}

For the coherent interface model, the displacements must be continuous at the interface whereas the strains must follow the Hadamard relation described in Eq. (15). These conditions can be enforced by superposing to the standard finite element field an enrichment term that possesses the above continuity conditions (XFEM method [21]). In this context, the approximation is defined at a particular point $\mathbf{x}$ lying in an element $\Omega_{e}$ by:

$$
\mathbf{u}^{h}(\mathbf{x})=\sum_{i=1}^{n} N_{i}(\mathbf{x}) \mathbf{u}_{i}+\sum_{j=1}^{m} N_{j}(\mathbf{x}) \psi(\mathbf{x}) \mathbf{a}_{j},
$$

where $N_{i}$ are the standard finite element shape functions associated with the nodes $n_{i}$ of the elements, $N_{j}(\mathbf{x})$ are the shape functions of the nodes of the elements whose support are cut by the interface (see figure 2) and $\psi(\mathbf{x})$ is a function with the required continuity. Due to the enrichment term in Eq. (33), the unknowns $\mathbf{u}_{i}$ become arbitrary coefficients, as well as the unknowns $\mathbf{a}_{j}$, and lose their kinematical meanings. To meet the Hadamard condition (15), we use the enriched approximation proposed in [29], which ensures good convergence properties, preserves the interpolant character of the approximation, 
and removes the issue of partially enriched elements [29]. Precisely, the enrichment function is defined by:

$\psi(\mathbf{x})=\sum_{i=1}^{n}\left|\phi_{i}\right| N_{i}(\mathbf{x})-\left|\sum_{i=1}^{n} \phi_{i} N_{i}(\mathbf{x})\right|$.

The numerical integration in the bulk is performed by using Gauss integration on subtriangles near the interface (see figure 3 ). The strain and stress tensors are then expressed in the vector forms,

$\boldsymbol{\epsilon}=\left[\begin{array}{l}\epsilon_{11} \\ \epsilon_{22} \\ \alpha \epsilon_{33} \\ 2 \epsilon_{12}\end{array}\right] \quad, \quad \boldsymbol{\sigma}=\left[\begin{array}{l}\sigma_{11} \\ \sigma_{22} \\ \alpha \sigma_{33} \\ \sigma_{12}\end{array}\right]$,

with $\alpha=0$ for plane strain problems and $\alpha=1$ for axisymmetric problems. In the foregoing, the indices 1,2 and 3 are associated with directions $\mathbf{e}_{x}, \mathbf{e}_{y}$ and $\mathbf{e}_{z}$ and $\mathbf{e}_{r}$, $\mathbf{e}_{z}$ and $\mathbf{e}_{\theta}$, according as a plane strain or an axisymmetric problem is concerned. The vector forms of surface strain and stress take the same form as in (35).

The specificity of the present problem is the presence of the internal virtual work term related to the implicit surface (not discretized by nodes) in Eq. (30). To evaluate the associated surface integral, we first approximate the interface by piece-wise linear segments in 2D (see figure 3 ). For this purpose, we first find the intersection between the level-set function $\phi(\mathbf{x})$ and the triangular mesh. The triangles cut by the interface $\Gamma_{I}$ are easily detected, as the values of $\phi$ evaluated at the two nodes of a given edge have opposite signs. The intersection between $\phi(\mathbf{x})=0$ and the edges of the mesh can then be approximated by using a linear interpolation of $\phi(\mathbf{x})$ based on the mesh. Once again, the linear interpolation is adopted, though higher-order approximation schemes could be used [28]. It is worth noting that despite the fact that the surface is implicit, its description accuracy depends on the local nodal density.

We then perform a Gauss integration on each edge of the 2D interface approximation. In the following examples, we have used three Gauss points in the triangles cut by the interface, one in the remaining triangles and two on each linear segment of the approximated interface. On substituting the trial and test functions from Eq. (33) in Eq. (30), and using the arbitrariness of nodal variations, the following discrete system of linear equations is obtained:

$\left(\mathbf{K}+\mathbf{K}^{s}\right) \mathbf{d}=\mathbf{f}$

where

$\mathbf{K}_{I J}=\int_{\Omega} \mathbf{B}_{I}^{T} \mathbf{C}^{(i)} \mathbf{B}_{J} \gamma d \Omega$

$\mathbf{f}=\int_{\Omega} \mathbf{N}^{T} \mathbf{b} \gamma d \Omega+\int_{\partial \Omega_{F}} \mathbf{N}^{T} \mathbf{F} \gamma d \Gamma+\int_{\Omega} \mathbf{B}^{T} \mathbf{C}^{(i)} \boldsymbol{\epsilon}^{*} \gamma d \Omega$

$+\int_{\partial \Gamma_{I}} \mathbf{N}^{T} \mathbf{P} \hat{\mathbf{F}} \gamma d l+\int_{\Gamma_{I}} \mathbf{B}^{T} \mathbf{M}_{p}^{T} \boldsymbol{\sigma}_{0} \gamma d \Gamma$ with $\gamma=1$ for plane strain problems and $\gamma=2 \pi r$ for axisymmetric problems. In the above equations, the matrix $\mathbf{B}_{I}$ is given by:

$\mathbf{B}_{I}=\left\{\begin{array}{ll}\frac{\partial \hat{N}_{I}(\mathbf{x})}{\partial x_{1}} & 0 \\ 0 & \frac{\partial \hat{N}_{I}(\mathbf{x})}{\partial x_{2}} \\ \alpha \frac{\hat{N}_{I}(\mathbf{x})}{x_{1}} & 0 \\ \frac{\partial \hat{N}_{I}(\mathbf{x})}{\partial x_{2}} & \frac{\partial \hat{N}_{I}(\mathbf{x})}{\partial x_{1}}\end{array}\right\}$,

where $\hat{N}_{I} \equiv N_{I}$ for a finite element displacement degree of freedom, and $\hat{N}_{I} \equiv \psi N_{I}$ for an enriched degree of freedom. In the above equations, $\mathbf{C}^{(i)}$ is the bulk stiffness matrix associated with the elasticity tensor of phase $i$. To determine the phase associated with a particular bulk integration point, we simply use the sign of $\phi(\mathbf{x})$ computed by the linear finite element discretization:

$\phi(\mathbf{x})=\sum_{i=1}^{n} N_{i}(\mathbf{x}) \phi_{i}$

The matrix $\mathbf{C}^{(i)}$ is defined according to:

$\mathbf{C}=\left[\begin{array}{cccc}(\lambda+2 \mu) & \lambda & \alpha \lambda & 0 \\ \lambda & (\lambda+2 \mu) & \alpha \lambda & 0 \\ \alpha \lambda & \alpha \lambda & \alpha(\lambda+2 \mu) & 0 \\ 0 & 0 & 0 & \mu\end{array}\right]$

where the superscripts $i$ have been omitted. The matrix $K^{s}$ is the interface rigidity matrix, expressed by:

$\mathbf{K}_{I J}^{s}=\int_{\Gamma_{I}} \mathbf{B}_{I}^{T} \mathbf{M}_{p}^{T} \mathbf{C}^{S} \mathbf{M}_{p} \mathbf{B}_{J} \gamma d \Gamma$

with $\mathbf{C}^{s}$ the surface stiffness matrix expressed by:

$\mathbf{C}^{s}=\left[\begin{array}{cc}\left(2 \mu_{s}+\lambda_{s}\right) P_{11}^{2} & \lambda_{s} P_{11} P_{22}+2 \mu_{s} P_{12}^{2} \\ \lambda_{s} P_{11} P_{22}+2 \mu_{s} P_{12}^{2} & \left(2 \mu_{s}+\lambda_{s}\right) P_{22}^{2} \\ \alpha \lambda_{s} P_{11} & \alpha \lambda_{s} P_{22} \\ \left(2 \mu_{s}+\lambda_{s}\right) P_{11} P_{12} & \left(2 \mu_{s}+\lambda_{s}\right) P_{12} P_{22}\end{array}\right.$

$\left.\begin{array}{cc}\alpha \lambda_{s} P_{11} & \left(2 \mu_{s}+\lambda_{s}\right) P_{11} P_{12} \\ \alpha \lambda_{s} P_{22} & \left(2 \mu_{s}+\lambda_{s}\right) P_{12} P_{22} \\ \alpha\left(2 \mu_{s}+\lambda_{s}\right) & \alpha \lambda_{s} P_{12} \\ \alpha \lambda_{s} P_{12} & \lambda_{s} P_{12}^{2}+\mu_{s}\left(P_{11} P_{22}+P_{12}^{2}\right)\end{array}\right]$.

The matrix $\mathbf{M}_{p}$ is constructed such that $\boldsymbol{\epsilon}_{s}=\mathbf{M}_{p} \boldsymbol{\epsilon}$ and is defined according to:

$\mathbf{M}_{p}=\left[\begin{array}{cccc}P_{11}^{2} & P_{12}^{2} & 0 & P_{11} P_{12} \\ P_{12}^{2} & P_{22}^{2} & 0 & P_{12} P_{22} \\ 0 & 0 & \alpha & 0 \\ 2 P_{11} P_{12} & 2 P_{12} P_{22} & 0 & \left(P_{12}^{2}+P_{11} P_{22}\right)\end{array}\right]$ 


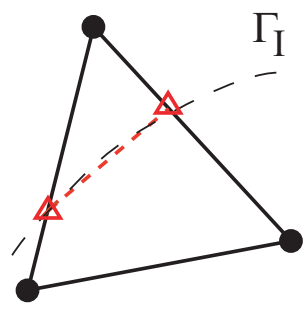

(a)

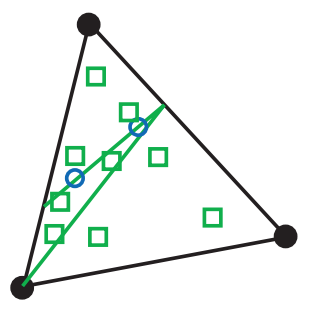

(b)
$\Delta$ Intersection between zero level-set function and the mesh

- Interface integration points

\section{Bulk integration points}

Fig. 3 (a) Approximated interface using interpolated level-set function on triangle edges; (b) triangulation of the elements cut by the interface and integration points.

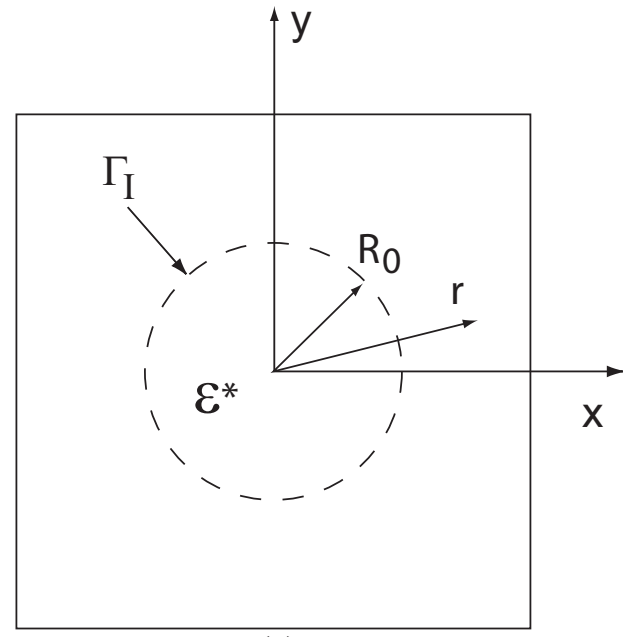

(a)

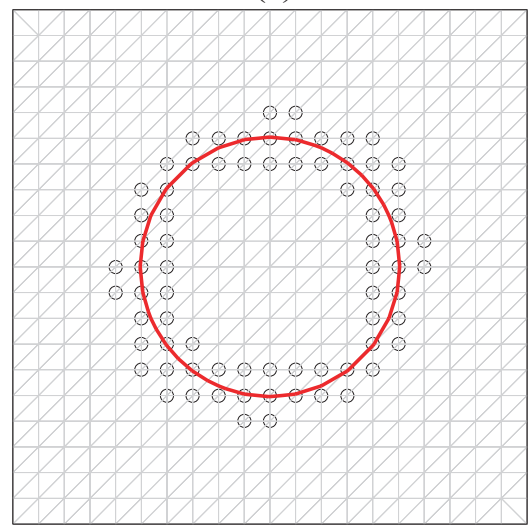

(b)

Fig. 4 First Eshelby problem model with imperfect coherent interface (a) Geometry; (b) Mesh, zero level set, and enriched nodes.

\section{Numerical examples}

5.1 Cylindrical inclusion under plane strain and with the coherent interface model

A cylindrical inclusion with an imperfect coherent interface in an infinite medium is submitted to a dilatational eigenstrain $\epsilon^{*}$. The exact strain solution of this problem is given in $[9 ; 13]$ by:

$$
\begin{aligned}
& \epsilon_{r r}(r)=\epsilon_{\theta \theta}(r)=A, \quad r<R_{0} \\
& \left\{\begin{array}{l}
\epsilon_{r r}(r)=-A \frac{R_{0}^{2}}{r^{2}}, \quad r>R_{0} \\
\epsilon_{\theta \theta}(r)=A \frac{R_{0}^{2}}{r^{2}}
\end{array}\right. \\
& A=\frac{3 K^{\prime} \epsilon^{*}-\tau_{0} / R_{0}}{2 \mu^{M}+3 K^{\prime} M+K^{\prime} S / R_{0}},
\end{aligned}
$$

where $R_{0}$ is the radius of the cylindrical inclusion (see figure 4$), \kappa^{\prime M}=2\left(\lambda^{M}+\mu^{M}\right) / 3, \kappa_{s}^{\prime}=\left(\lambda^{S}+2 \mu^{S}\right)$ is the plane strain surface modulus, and $\epsilon^{*}$ is a prescribed dilatational eigenstrain in cylindrical inclusion with $\epsilon^{*}=$ $\epsilon_{11}^{*}=\epsilon_{22}^{*}, \epsilon_{33}^{*}=0$.

By using the kinematical relations $\epsilon_{r r}=\partial u_{r} / \partial r$ and $\epsilon_{\theta \theta}=u_{r} / r$ we obtain the displacement field as:

$u_{r}(r)= \begin{cases}A r & , \quad 0 \leq r \leq R_{0} \\ A \frac{R_{0}^{2}}{r}, & r \geq R_{0} .\end{cases}$

We consider an axisymmetric model in a finite square domain by imposing the exact displacement solution on the external boundary. Then, the interface does not coincide with the mesh. To examine the size effect, we propose the following indicator:

$$
\begin{aligned}
& \beta=\frac{\left|\int_{\Gamma_{I}} \boldsymbol{\sigma}_{s}(\mathbf{u}): \boldsymbol{\epsilon}_{s}(\mathbf{u}) d \Gamma\right|}{\left|\int_{\Gamma_{I}} \boldsymbol{\sigma}_{s}(\mathbf{u}): \boldsymbol{\epsilon}_{s}(\mathbf{u}) d \Gamma\right|+\left|\int_{\Omega} \boldsymbol{\sigma}(\mathbf{u}): \boldsymbol{\epsilon}(\mathbf{u}) d \Omega\right|} \\
& =\frac{\left|E_{s}\right|}{\left|E_{s}\right|+\left|E_{b}\right|},
\end{aligned}
$$

where $E_{s}$ denotes the surface energy and $E_{b}$ stands for the bulk energy. The following numerical parameters were used for the bulk material : $E=3 \mathrm{GPa}, \nu=0.3, \epsilon^{*}=0.5$, $\tau_{0}=0$. A regular mesh of $40 \times 40$ nodes is used. The surface parameters can be obtained through molecular dynamics computations. Here, we use those obtained by Miller and Shenoy in [1]: (a) $\lambda_{s}=6.842 \mathrm{~N} / \mathrm{m}, \mu_{s}=$ $-0.375 \mathrm{~N} / \mathrm{m}$, which gives a positive $\kappa_{s}^{\prime}=6.091 \mathrm{~N} / \mathrm{m}$, (b) $\lambda_{s}=3.48912 \mathrm{~N} / \mathrm{m}, \mu_{s}=-6.2178 \mathrm{~N} / \mathrm{m}$, which gives 


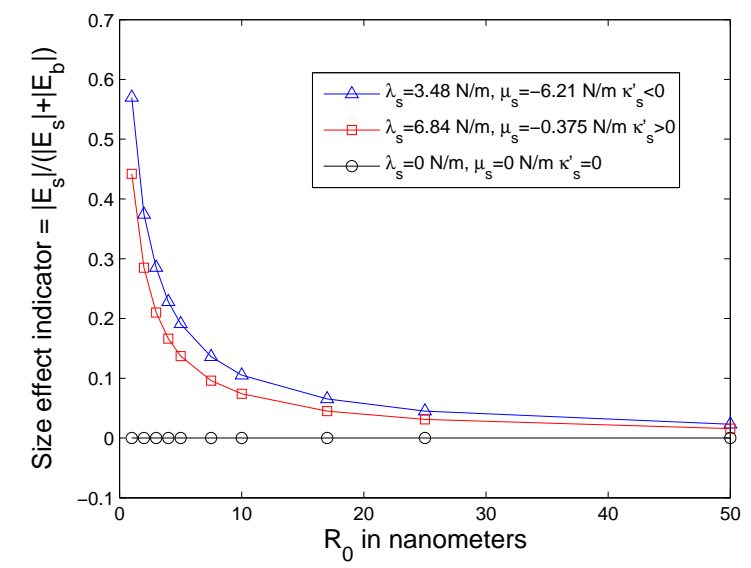

Fig. 5 Size effect indicator versus inclusion radius.

a negative plane-strain surface modulus $\kappa_{s}^{\prime}=-8.94948$ $\mathrm{N} / \mathrm{m}$, and (c) the classical case where $\lambda_{s}=0 \mathrm{~N} / \mathrm{m}$, $\mu_{s}=0 \mathrm{~N} / \mathrm{m}, \kappa_{s}^{\prime}=0 \mathrm{~N} / \mathrm{m}$.

As noted by Shenoy in [30], the surface elastic tensor $C_{i j k l}^{s}$ needs not to be positive definite, i.e. the quadratic form $C_{i j k l}^{s} \epsilon_{i j}^{s} \epsilon_{k l}^{s}$ need not to be non-negative. At first glance it may suggest a violation of basic thermodynamic postulates. It must be noted that positive definiteness of the bulk elastic modulus tensor which guarantees the stability of the solid cannot be applied to the surface elastic tensor. This is due to the fact that a surface cannot exist independently of the bulk, and only the total energy (bulk+surface) needs to satisfy the positive definiteness condition.

As seen from figure 5, the presence of the surface term on the left-hand of Eq. (26) introduces size effects, when the internal surface energy surface becomes important compared to the internal volume energy.

Next we compare the exact solution with the solution computed from the proposed numerical approach for $f=$ 0.2 . For a fair comparison between the different sets of parameters, we chose the inclusion radius $R_{0}$ in each simulation such as $\beta=0.4$. Thus for the set (a), we chose $R_{0}=1 \mathrm{~nm}$, and for the example (b) we chose $R_{0}=1.8$ $\mathrm{nm}$. Computations are performed for different regular triangular meshes with increasing nodal density, ranging from $10 \times 10$ nodes to $80 \times 80$ nodes. The convergence results about the relative energy norm

$$
\begin{aligned}
& \frac{\left\|\mathbf{u}^{h}(\mathbf{x})-\mathbf{u}(\mathbf{x})\right\|_{E(\Omega)}}{\|\mathbf{u}(\mathbf{x})\|_{E(\Omega)}} \\
& =\frac{\left(\int_{\Omega}\left(\boldsymbol{\epsilon}^{h}(\mathbf{x})-\boldsymbol{\epsilon}(\mathbf{x})\right): \mathbb{C}^{(i)}:\left(\boldsymbol{\epsilon}^{h}(\mathbf{x})-\boldsymbol{\epsilon}(\mathbf{x})\right)\right)^{\frac{1}{2}}}{\left(\int_{\Omega} \boldsymbol{\epsilon}(\mathbf{x}): \mathbb{C}^{(i)}: \boldsymbol{\epsilon}(\mathbf{x})\right)^{\frac{1}{2}}}
\end{aligned}
$$

are reported in figure 6 .

For $\kappa_{s}^{\prime}=0$ (no surface effects), an expected rate of convergence $r \approx 1$ is appreciated. For $\kappa_{s}^{\prime}<0$ and $\kappa_{s}^{\prime}>0$, the proposed approach leads to a convergent solution,

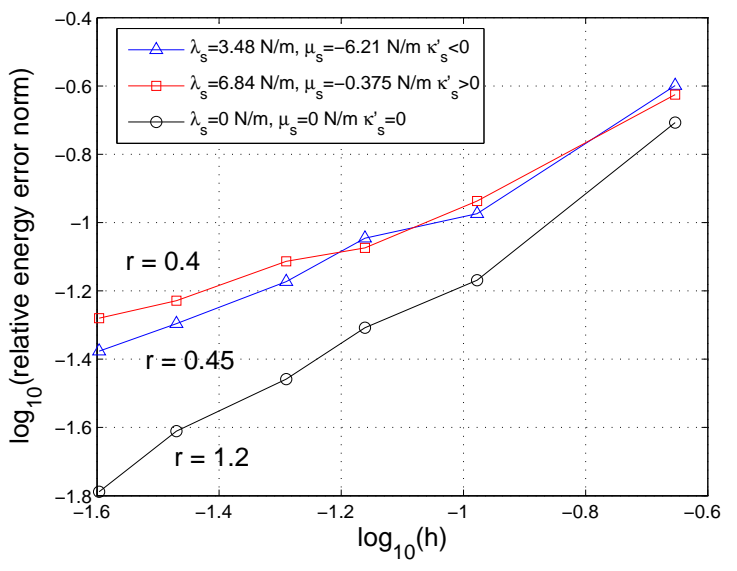

Fig. 6 Convergence analysis for the cylindrical inclusion problem with the coherent interface model.

but without an optimal rate of convergence. Though it is adequate for most purposes, it indicates that there is room for improvement of the method.

It is worth pointing out that for $\kappa_{s}^{\prime}<0$, the contribution of the negative-definite surface stiffness matrix $\mathbf{K}_{s}$ in (36) influences the condition number of the total stiffness matrix, which may consequently tend to be ill conditioned. This issue has also been reported by Gao et al. in [18]. When the ill condition leads to inaccurate results, special numerical methods should be applied to improve the precision of solutions. In the present work, we have used an iterative solver, the biconjugate gradients methods [31] (bicg in Matlab).

5.2 Size-dependent overall properties of a material with cylindrical nanovoids

In this example, we compute the effective bulk modulus of an aluminium material containing nano voids. For this purpose, we perform numerical linear homogenization on a RVE containing a coherent interface whose geometry is depicted in figure 4 (b). For a constant volume fraction, we vary the void radius and compute for each size the effective bulk modulus. Estimated effective properties for long cylindrical nanofibers with coherent interfaces have been provided in [15]. We then compare the results obtained by the present XFEM approach with the reference normalized effective bulk modulus $\kappa^{\text {eff }} / \kappa_{M}, \kappa_{M}$ being the matrix bulk modulus. In this example, we have used a regular 80 nodes grid. The material parameters of the matrix are $E=70 \mathrm{MPa}$ and $\nu=0.32$. The results are depicted in figure 7 for a volume fraction $f=0.2$. Good agreement between the reference solution and the numerical computations is noticed.

We obtain the expected effects, i.e. the effective properties are not sensitive to the nanovoid radius value for relatively large sizes, but the values of effective bulk modulus clearly deviate for small sizes of nanovoids. 


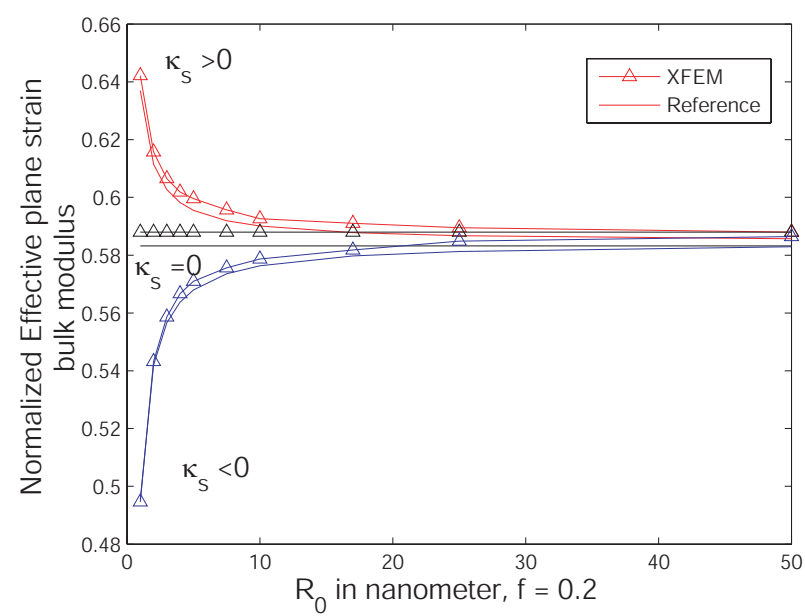

Fig. 7 Size-dependent effective plane-strain modulus with surface effects versus void radius, $f=0.2$.

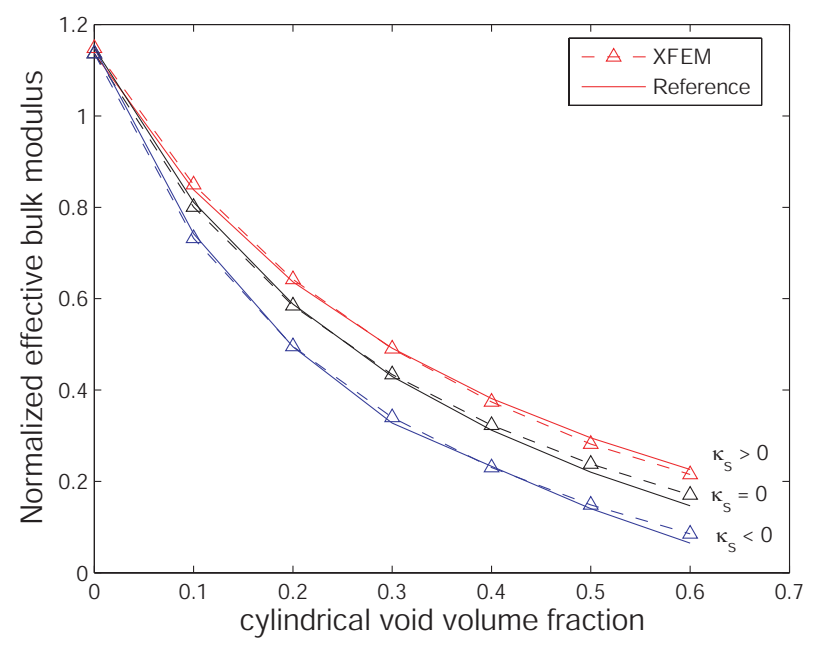

Fig. 8 plane-strain bulk modulus for $R=1 \mathrm{~nm}$ versus void volume fraction.

Now for a fixed radius $R=1 \mathrm{~nm}$ (at which size effects are predominant for the used set of parameters), we vary the volume fraction by changing the size of the enclosing square domain. Due to the implicit description of the interface, generating different geometries only requires changing the equation describing the level set, and does not involve any mesh generation. We compute the effective properties of the RVE using the aforementioned approach for volume fractions ranging from 0 (no interface) to 0.6. The results comparing the present XFEM approach and the reference solution computed from [15] are provided in figure 8 . Very good agreement between theory and numerical solutions is noticed, and the surface effects are clearly shown up.

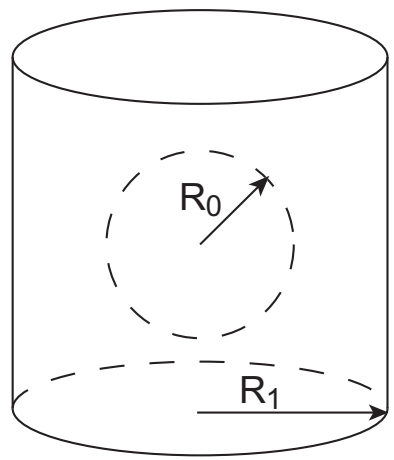

(a)

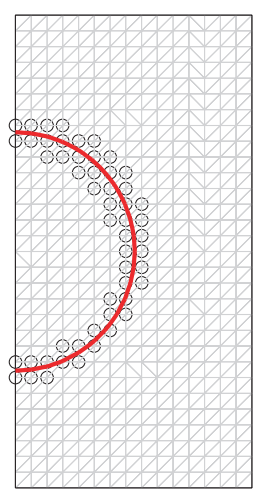

(b)
Fig. 9 Spherical inclusion with imperfect interface; (a) geometry; (b) finite axisymmetric domain, zero level-set and enriched nodes.

\subsection{Spherical inclusion}

Here we test the proposed XFEM methodology through an axisymmetric problem. A spherical inclusion with an imperfect coherent interface in an infinite medium is submitted to a dilatational eigenstrain $\epsilon^{*}$. We model the spherical inclusion using axisymmetric triangular elements (see figure 9). For this problem, the exact solution is given by $[10 ; 14]$ :

$$
\begin{aligned}
& \epsilon_{r r}(r)=\epsilon_{\theta \theta}=\epsilon_{\phi \phi}(r)=A, \quad r<R_{0} \\
& \left\{\begin{array}{l}
\epsilon_{r r}(r)=-2 A \frac{R_{0}^{3}}{r^{3}} \\
\epsilon_{\theta \theta}(r)=\epsilon_{\phi \phi}(r)=A \frac{R_{0}^{3}}{r^{3}}
\end{array}, \quad r>R_{0}\right. \\
& A=\frac{3 \kappa^{M} \epsilon^{*}-2 \tau_{0} / R_{0}}{4 \mu^{M}+3 \kappa^{M}+2 \kappa^{S} / R_{0}},
\end{aligned}
$$

where $\kappa^{M}=\lambda^{M}+2 \mu^{M} / 3, \kappa^{S}=2\left(\lambda^{S}+\mu^{S}\right), \epsilon^{*}=\epsilon_{11}^{*}=$ $\epsilon_{22}^{*}=\epsilon_{33}^{*}$. The displacement field is given by:

$u_{r}(r)= \begin{cases}A r & , \quad 0 \leq r \leq R_{0} \\ A \frac{R_{0}^{3}}{r^{2}}, & r \geq R_{0} .\end{cases}$

We chose $E=10 \mathrm{MPa}, \nu=0.3$ and $\epsilon^{*}=0.5$, and $\tau=0$. The radius is set as $R_{0}=1 \mathrm{~nm}$, and the radius of the cylindrical box $R_{1}$ (see figure 9 ) is fixed such that a given volume fraction $f=0.2$ is met, according to $R_{1}=\left(\sqrt{\pi R_{0}^{2} / f}\right) / 2$. For these parameters, the surface effects indicator has the value $\beta=0.4$. Symmetry conditions are applied on the boundary $r=0$ and exact displacement solution is imposed on the external boundary of the domain. Convergence results in energy norm error for $\kappa_{s}=0, \kappa_{s}>0$, and $\kappa_{s}<0$ using the same surface parameters as in previous examples are presented in figure 10. The indicated rate of convergence is the one of a linear polynomial fit on the last four point of the curve. Here again, We note that the XFEM solution is convergent, though not at an optimal rate. When no surface 


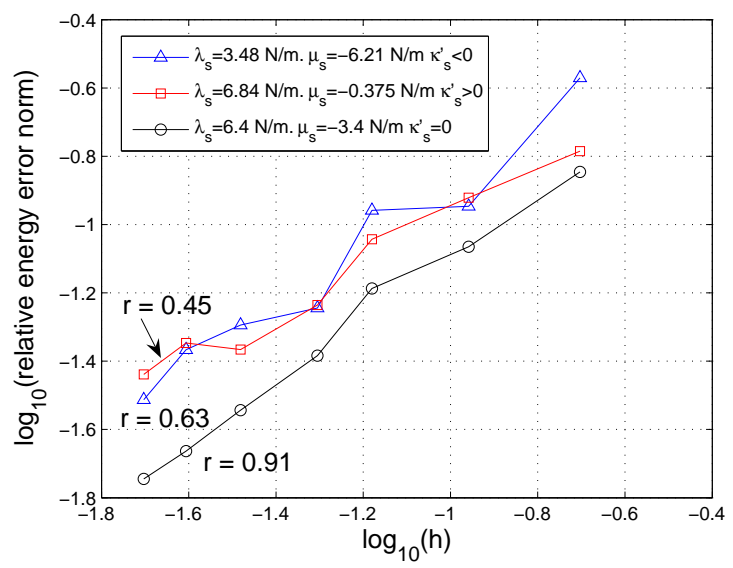

Fig. 10 Convergence analysis for the spherical inclusion problem with stiff coherent imperfect interface.

effects occur $\left(\kappa_{s}=0\right)$, the computed solution converges to the exact one at the expected rate of convergence.

For spherical void with a coherent surface, the effective modulus can be evaluated according to [12]:

$$
\begin{aligned}
& \kappa^{e f f}=\frac{3 \kappa^{I}\left(3 \kappa^{M}+4 f \mu^{M}\right)}{3\left[3(1-f) \kappa^{I}+3 f \kappa^{M}+2 \mu^{M}\left(2+\kappa_{r}^{S}-f \kappa_{r}^{S}\right)\right]} \\
& +\frac{2 \mu^{M}\left[4 f \mu^{M} \kappa_{r}^{S}+3 \kappa^{M}\left(2-2 f+\kappa_{r}^{S}\right)\right]}{3\left[3(1-f) \kappa^{I}+3 f \kappa^{M}+2 \mu^{M}\left(2+\kappa_{r}^{S}-f \kappa_{r}^{S}\right)\right]},
\end{aligned}
$$

with $\kappa_{r}^{S}=\kappa^{S} /\left(R_{0} \mu^{M}\right)$, the superscripts $M$ and $I$ denoting the matrix and inhomogeneity, respectively.

In figure 11 we compare the normalized effective reference bulk modulus $\kappa^{e f f} / \kappa_{M}$ for $f=0.5$ for different radii of the spherical void. In figure 12 we compare the normalized effective reference bulk modulus for a fixed radius $R_{0}=1 \mathrm{~nm}$ while varying the volume fraction $f$. Excellent agreement with the reference solution is noticed and here again the surface effects are clearly shown.

\subsection{Random nanostructure}

Next we explore the effective properties of aluminium containing randomly distributed nanopores with constant radii, in order to investigate its size-effects on effective properties. For this purpose, we use 30 circular voids randomly distributed, by choosing the size of the square domain such that the volume fraction is $f=0.3$, and we vary the radius of the pores. A uniform mesh of $80 \times 80$ nodes is used, and the level-set function was defined according to

$$
\phi(\mathbf{x})=\min _{\mathbf{x}_{c}^{i} \in \Omega_{c}^{i}}\left\{\left\|\mathbf{x}-\mathbf{x}_{i}^{c}\right\|-r_{c}^{i}\right\}, \quad i=1,2, \ldots, n_{c},
$$

where $\Omega_{c}^{i}$ is the domain of the $i$ th void, $n_{c}$ is the number of circular voids, and $\mathbf{x}_{c}^{i}$ and $r_{c}^{i}$ are the center and radius of the $i$ th void, respectively.

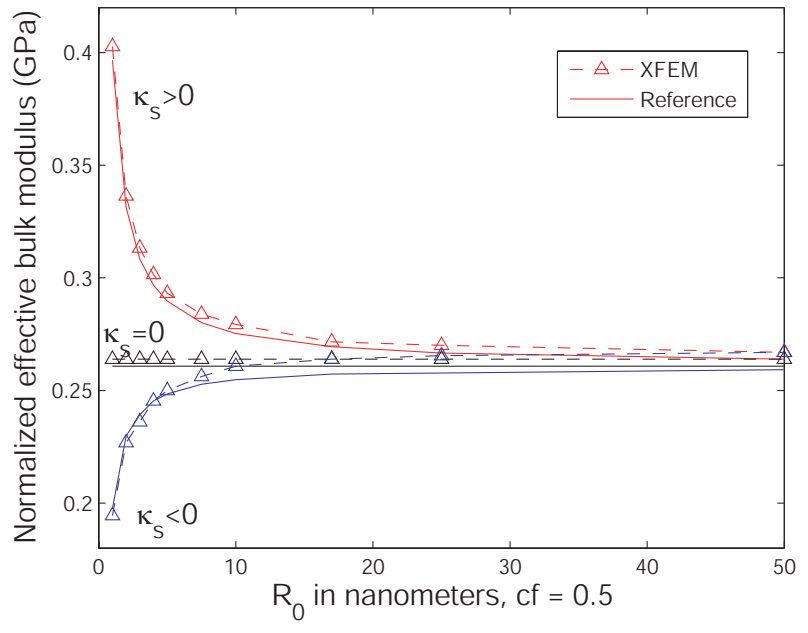

Fig. 11 Normalized effective bulk modulus for different spherical void radius.

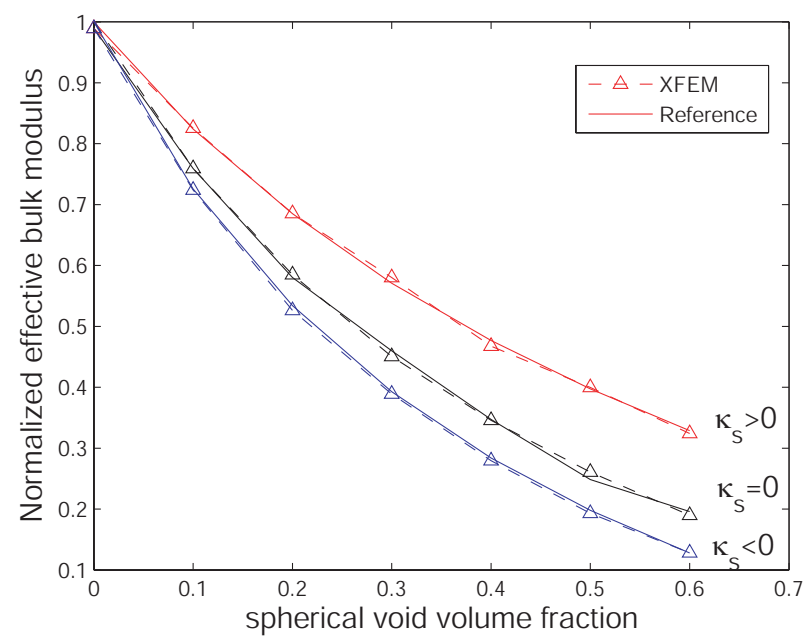

Fig. 12 Normalized effective bulk modulus for different void volume fractions, $R_{0}=1 \mathrm{~nm}$.

Different distributions are shown in figure 13. Note that avoiding the construction of a conforming mesh for each generation of a random microstructure by means of the level set technique greatly simplifies the analysis.

For each radius of nanopore, we generate random microstructures and compute the homogenized bulk modulus until we have reached statistical convergence on the mean value of the effective bulk modulus. Examples of statistical convergence are shown in figure 14 . The results for the effective bulk modulus are presented in figure 15. The size effects with different nanovoid radii can be clearly observed.

\subsection{Shape of nano inclusion}

In this example, we investigate the influence of the shapes of nanovoid on the effective properties and their possible 

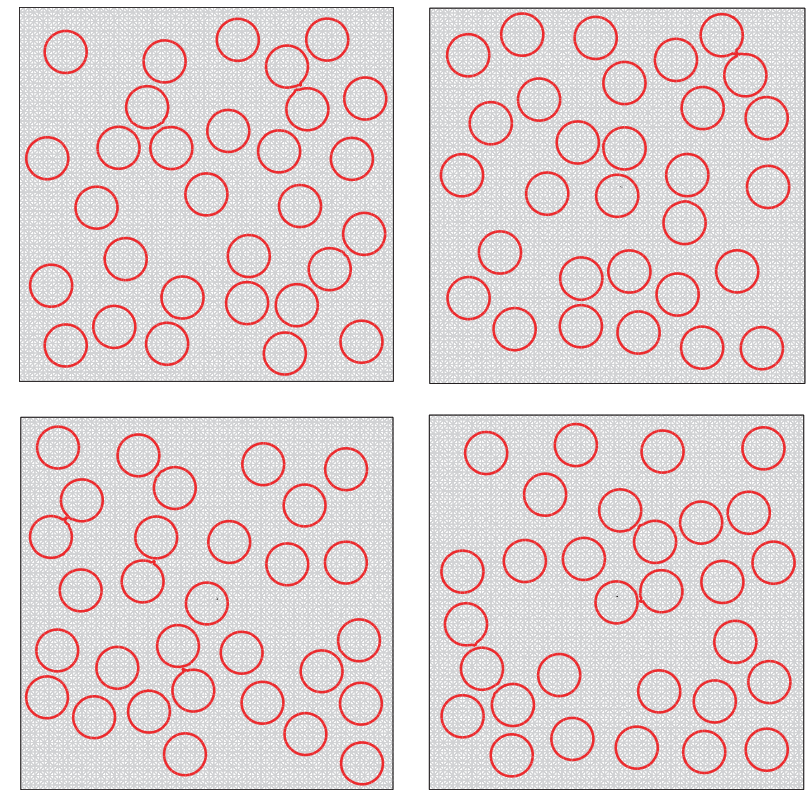

Fig. 13 Level set function for the different randomly distributed nanopores.

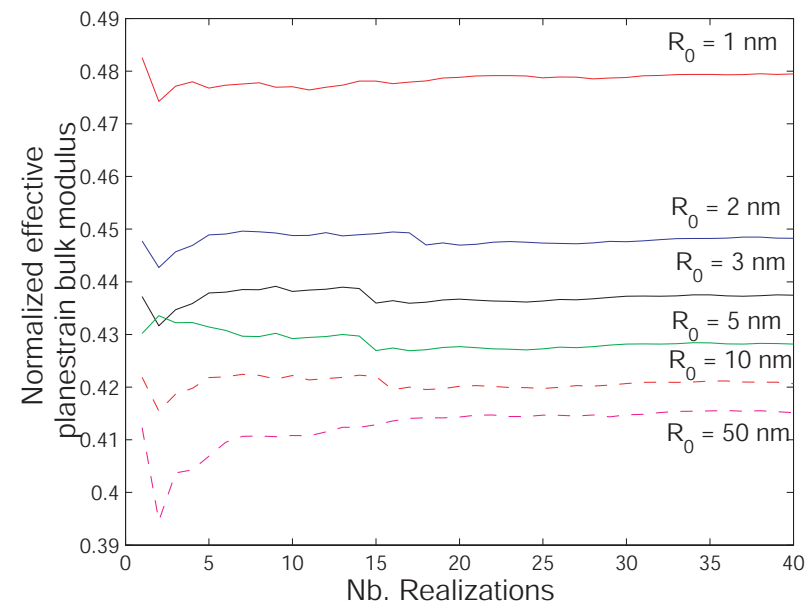

Fig. 14 Statistical convergence of the effective bulk modulus for various void radii.

influence on the size effects. For this purpose, different shapes are generated, as depicted in figure 16.

With the proposed procedure, it is easy to introduce an imperfect interface for an arbitrary geometry, without requiring any surface elements. In figure 17, we present the effective bulk moduli associated with the different shapes, for different sizes. For each shape, we have chosen the size parameters such as its volume is equal to the one of the circular void. For polygonal shapes, the level-set function was constructed using Eq. (56), and replacing $r_{c}$ by the distance between the center $x_{i}^{c}$ and $x_{I}$ defined as the intersection between $\left\{x_{i}^{c}-\mathbf{x}\right\}$ and the boundary of the polygonal shape. For the non-convex shapes, we

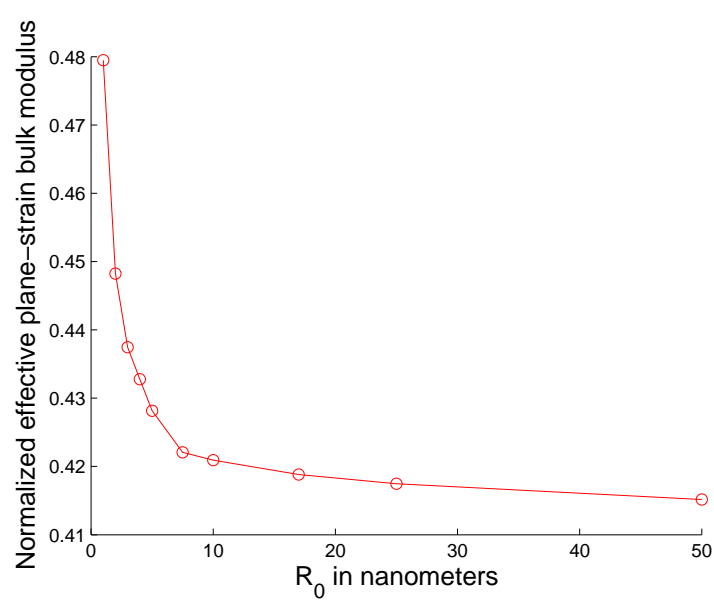

Fig. 15 Size effects in bulk modulus for randomly distributed nanopores.
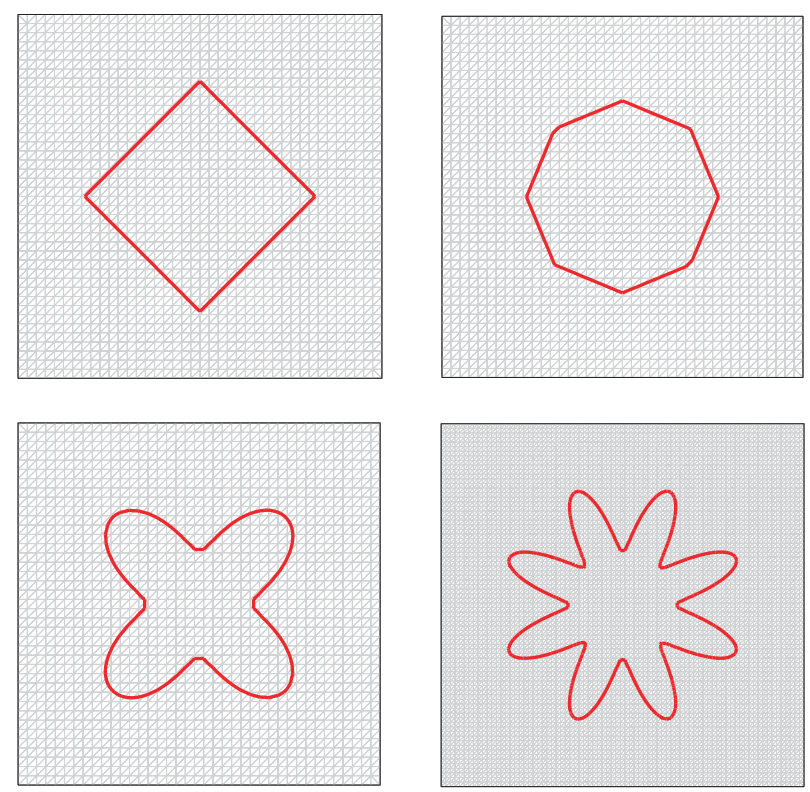

Fig. 16 Zero level-set function of different shapes of nanostructures with same volume fractions $f=0.2$.

have used the following function describing their radius in cylindrical coordinates:

$$
R(\theta)=R_{0}+A \sin (B \theta)
$$

where $R_{0}$ is the reference radius, $B$ denotes the number of oscillations and $A$ is the amplitude of oscillations. In the example, we have chosen $B=4$ and $B=8$, and $A=0.4 R_{0}$.

The associated volume area can be derived by:

$$
\begin{aligned}
& V=\frac{4 A^{2} B \pi+8 A R_{0}+8 B \pi R_{0}^{2}-8 A R_{0} \cos (2 B \pi)}{8 B} \\
& +\frac{A^{2} \sin (4 B \pi)}{8 B}
\end{aligned}
$$




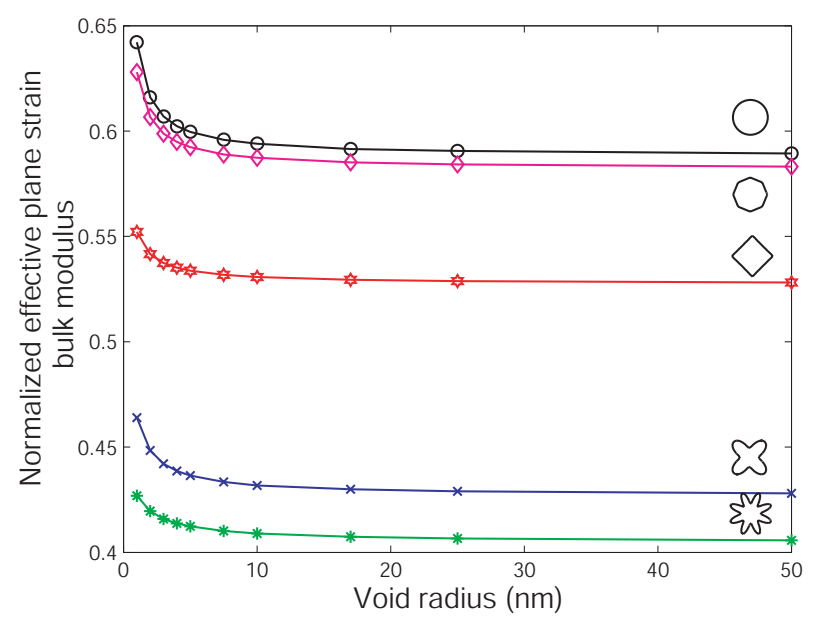

Fig. 17 Size and shape dependent properties of the nanostructures.

which allows to chose the size of the square box in order to meet the desired volume fraction, which here is chosen to be $f=0.2$.

Results for the normalized effective bulk modulus are reported in figure 17 . We notice that size effects occur independently of the shape of the nanostructures. As expected, we note that the shape greatly influences the effective bulk modulus. The circular shape offers the best stiffness against compression, while the non convex shapes do not resist well to compression.

\section{Conclusion}

In this work, a numerical procedure has been proposed to compute the overall elastic properties of nanomaterials and nanostructures with surface/interface effects. For this purpose, the coherent interface model has been adopted, leading to an additional stiffness matrix. Since the ratio between the surface and bulk strain energies is not preserved when the volume fraction of the nanoinhomogeneities in a material remains fixed but their sizes or/and shapes change, the effective properties of the material depend on the sizes and the shapes of the nanoinhomogeneities. To handle efficiently complex and arbitrary nano-inhomogeneities only through regular meshes, we have developed a level-set approach in tandem with an extended finite element method. This approach allows the generation of complex microstructures in an automatic manner without the burden of meshing, and hence constitutes an efficient numerical tool for analyzing highly inhomogeneous materials. The proposed $\mathrm{XFEM} /$ level set approach has been validated in the $2 \mathrm{D}$ context by applying it to different problems with known exact analytical solutions. The proposed approach has also been employed to determine the effective elastic moduli of materials with randomly distributed nanopores. As expected, the effective elastic moduli are not only size-dependent but also shape-dependent with respect to nanopores.

The implementation and application of the proposed XFEM/level set approach in the three-dimensional context are in progress. Note that this approach can also be extended to other mechanical and physical phenomena involving imperfect interfaces.

\section{Aknowledgements}

The support this work enjoys from Electricité De France (EDF) is gratefully acknowledged. The authors thank profs. C. Desceliers and C. Soize (Université Paris-Est, Marne-la-Vallée) for their advices on iterative solvers that are used in this work for solving the ill-conditioned problems.

\section{References}

1. Miller RE, Shenevoy VB (2000) Size-dependent elastic properties of nanosized structural elements. Nanotechnology. 11(3):139-147

2. Haile JM, (1992) Molecular Dynamics Simulation, Wiley, New York.

3. Hoover WG (1986) Molecular Dynamics, Springer-Verlag, Berlin.

4. Rapaport DC (2004) The Art of Molecular Dynamics Simulation, Cambridge University Press, New York.

5. Povstenko YZ (1993), Theoretical investigation of phenomena caused by heterogeneous surface tension in solids, J. Mech. Phys. Solids 41:1499-1514

6. Hadal R, Yuan Q, Jog JP, Misra RDK (2006), On stress whitening during surface deformation in clay-containing polymer nanocomposites: A microstructural approach. Mater. Sci. Eng. A 418:268-281

7. Benveniste Y (2006) A general interface model for a threedimensional curved thin anisotropic interphase between two anisotropic media. J. Mech. Phys. Solids 54:708-734

8. Gurtin ME, Weissmuller J, Larche F (1998) A general theory of curved deformable interfaces in solids at equilibrium. Philos. Mag. A 78(5):1093-1109

9. Sharma P, Ganti S (2006) Size-dependent Eshelby's tensor for embedded nano-inclusions incorporating surface/interface energies. J. Appl. Mech.-Trans. ASME 71(5):663-671

10. Sharma P, Ganti S, Bathe N (2003) Effect of surfaces on the size-dependent elastic state of nano-inhomogeneities. Appl. Phys. Lett. 82(4):535-537

11. Chen T, Dvorak GJ, Yu CC (2007) Solids containing spherical nano-inclusions with interface stresses: Effective properties and thermal-mechanical connections. Int. J. Solids Struct. 2007 44(3-4):941-955

12. Duan HL, Wang J, Huang ZP, Karihaloo BL (2005) Sizedependent effective elastic constants of solids containing nano-inhomogeneities with interface stress. J. Mech. Phys. Solids 53(7):1574-1596

13. Sharma P, Ganti S (2005) Erratum: "Size-dependent Eshelby's tensor for embedded nano-inclusions incorporating surface/interface energies"' [Journal of Applied Mechanics, 2004, 71(5), pp. 663-671]. J. Appl. Mech.-Trans. ASME 72:628

14. Sharma P. Ganti S. Bathe N (2006) Erratum: "Effect of surfaces on the size-dependent elastic state of nano- 
inhomogeneities (vol 82, pg 535, 2003). Appl. Phys. Lett. 89(4): No. 049901

15. Le Quang H, He Q-C (submitted) Estimation of the imperfect thermoelastic moduli of fibrous nanocomposites with cylindrically anisotropic phases. Submitted to Arch. of Appl. Mech.

16. Le Quang H, He Q-C, Size-dependent effective thermoelastic properties of nanocomposites with spherically anisotropic phases (2007) J. Mech. Phys. Solids 55(9):1889-1921

17. Dingreville R, Qu J, Cherkaoui M (2005) Surface free energy and its effect on the elastic behavior of nano-sized particles. J. Mech. Phys. Solids 53(8):1827-1854

18. Gao W, Yu S, Huang G (2006) Finite element characterization of the size-dependent mechanical behaviour in nanosystems. Nanotechnology 17:1118-1122

19. Osher S, Sethian JA (1998) Fronts propagating with curvature-dependent speed: Algorithms based on Hamilton-Jacobi formulations. J. Comput. Phys. 79(1):12-49

20. Belytschko T, Black T (1999) Elastic crack growth in finite elements with minimal remeshing. Int. J. Numer. Methods Eng. 45(5):601-620

21. Moës N, Dolbow J, Belytschko T (1999) A finite element method for crack growth without remeshing. Int. J. Numer. Methods Eng. 46(1):131-156

22. Sukumar N,Chopp DL, Moës N, Belytschko T (2001) Modeling holes and inclusions by level sets in the extended finite-element method. Comput. Meth. Appl. Mech. Eng. 190:6183-6200

23. Sukumar N, Huang ZY, Prevost J-H, Suo Z, (2004) Partition of unity enrichment for bimaterial interface cracks. Int. J. Numer. Methods Eng. 59:1075-1102

24. Do Carmo MP (1976) Differential Geometry of Curves and Surfaces. Prentice-Hall, New-Jersey.

25. Thorpe JA (1979) Elementary Topics in Differential Geometry. Springer-Verlag, Berlin, 1979.

26. Cammarata RC (1994) Surface and interface stress effects in thin films. Prog. in Surf. Sci. 46:1-38

27. Chessa J, Belytschko T (2003) An enriched finite element method and level sets for axisymetric two-phase flow with surface tension. Int. J. Numer. Methods Eng. 58:2041-2064

28. Belytchko T, Parimi C, Moës N, Sukumar N, Usui S (2003) Structured extended finite element method for solids defined by implicit surfaces. Int. J. Numer. Methods Eng. 56: 609-635

29. Moës N, Cloirec M, Cartraud P, Remacle J-F (2003) A computational approach to handle complex microstructure geometries. Comput. Meth. Appl. Mech. Eng. 192:3163-3177

30. Shenoy VB (2005) Atomistic calculations of elastic properties of metallic fcc crystal surfaces. Phys. Rev. B 71:094104

31. Barrett R, Berry TF, Chan et al. (1994) Templates for the solution of linear systems: building blocks for iterative methods. SIAM, Philadelphia, 1994. 